and his associates; this leads one to expect that the structure will be essentially the same, despite the large differences in sequence. The residues known to be parts of the active centre remain in place, and the overall charge is also essentially unchanged. Most of the substitutions are in fact at the surface of the molecule: in the closely packed hydrophobic interior, the steric restrictions are exacting. Among the substitutions are three new proline residues, none happily in $a$-helical sections of the chain. A partial sequence of horse pancreatic ribonuclease is also presented, and shows considerable differences from the other two.

The rat enzyme has three extra residues at the $N$. terminal end, and the $S$-peptide region is therefore enlarged to 23 , as well as differing in no fewer than 10 of the other 20 residues from bovine $S$-peptide. (The $S$-peptide, it will be recalled, is the terminal segment which can be removed after proteolytic fission of one bond, with complete loss of activity: if it is returned to the truncated enzyme, it attaches non-covalently, with full restoration of activity.) This is interesting, in view of recent work on synthesis of S-peptide analogues. It is known that six residues can be excised from the $S$-peptide without detriment to its function. Finn and Hofmann (J. Amer. Chem. Soc., 89, 5298; 1967) have now shown that elimination of his-12part of the active centre-from the $S$-peptide leads to loss of reactivity, but hardly at all of affinity for the residual $S$-protein. The sites which are vital for attachment to $S$-protein, rather than activity, are glu-2, asp-14 and met-13, and these may not be tampered with. All these, as expected, are unchanged in the rat enzyme, whereas all six inessential residues (15-20) are changed. Scoffone et al. (ibid., 5450) have also synthesized $S$-peptide analogues: arg-10 has been replaced by ornithine, and phe-8 by tyrosine, isoleucine or alanine. In the last two cases binding is diminished, but tyrosine replaces phenylalanine with hardly any disadvantage. This is curious, for phe-8 is an internal residue, so the usual steric exclusivity is violated. Even in the other two eases, activity remains when a large excess of peptide is added to induce a measure of binding.

Yet another fascinating observation is that of Neumann et al. (Europ. J. Biochem., 3, 171; 1967), who have achieved partial reduction of pancreatic ribonuclease with a new reagent, phosphorothioate. In general, the four disulphide bonds are protected in the native state, but with this reagent two are cleaved in aqueous solution. The broken cystines have been identified by peptide mapping. As in one or two other cases recently reported, the partly cleaved ribonuclease retains its native conformation by all criteria. Thus it is immunologically identical with the native protein, and the three buried tyrosines remain non-titratable, and are exposed only on denaturation. In particular, the activity towards RNA is fully intact, and the activity towards cytidine cyclic phosphate is actually enhanced more than two-fold-it must be supposed by virtue of increased flexibility. The two surviving cystines are essentially "internal" residues. The susceptibility of the protein to attack by trypsin is much increased, which suggests that a general loosening of the structure can be tolerated, and that the steric limitations for activity are quite modest in large tracts of the sequence, as the wholesale substitutions in the rat enzyme also indicate.

\section{Model Cell Membranes}

\section{from a Correspondent}

ARTIFicial cell membranes, less than $100 \AA$ thick, can be prepared by allowing a film of lipid to form across a hole in a polythene cup separating two aqueous phases. Increasingly, these preparations are being used as model systems to test particular hypotheses in membrane transport. Thus, Tosteson and his colleagues (Andreoli et al., J. Gen. Physiol., 50, 1729; 1967) have been trying for a number of years to define the molecular basis of the difference between the HK (high potassium) and LK (low potassium) strains of sheep, where potassium refers to the concentration of this ion in the red blood cells of these animals. Transport studies have shown that the two strains differ in the ionic permeabilities of their cell membranes; the HK sheep are sodium selective, and the LK sheep prefer potassium ions. In the present paper, the lipids have been isolated from the cell membranes, artificial membranes prepared from these lipids and the permeability of such membranes measured. It is disappointing that artificial membranes from the two strains of sheep differ in no significant way; the artificial membranes do not distinguish between sodium and potassium and are also more permeable to these ions than to chloride. In contrast, the intact cell, for both strains, has a chloride permeability some $10^{6}$ times greater than that for cations. The difference between intact cells and model membranes seems to lie in the contribution of the membrane protein, and it is here that the gene distinguishing the HK and LK strains must act.

Another example of the impact of model membrane studies comes from Cass and Finkelstein (J. Gen. Physiol., 50, 1765; 1967), who have compared the water permeability of thin lipid films, when water movement occurs by diffusion (using isotopically labelled water) or by hydrodynamic flow (on setting up an osmotic gradient across the membrane). Studies of cell membranes have often shown that these two permeability coefficients differ. Because hydrodynamic flow across porous membranes, but not diffusion flow, is affected by the pore size, results such as these suggest that water moves through cell membranes along narrow channels. It was very surprising, therefore, that early studies on the artificial lipid membranepresumably a continuous bilayer-showed a difference between the two permeability coefficients. Cass and Finkelstein now show that the early results were almost certainly caused by the presence of unstirred layers of water in the diffusion experiments and that for the lipid bilayers the diffusion and hydrodynamic permeabilities for water do not differ. Hence water movement most probably occurs after dissolution in the lipid. It is pertinent to recall, therefore, Dainty's suggestion that diffusion studies on intact cells may be in error because of the presence of unstirred layers. For the human erythrocyte, however, where the two permeability eoefficients differ by a factor of $2 \cdot 4$, an elegant study (Sha'afi et al., J. Gen. Physiol., 50, 1377 ; 1967) has shown that the unstirred layer is only $5 \cdot 5 \mu$ thick, and that the effect of this layer is insignificant. Valuable as the studies on artificial lipid membranes may be, therefore, they strongly suggest that membrane proteins play at least as important a part as lipid in determining the permeability properties of cell membranes. 